\title{
Matrices of Spin-Orbit Interaction of the Electron Configuration $d^{4} \mathrm{~s}$
}

\author{
W. R. Bozman and R. E. Trees
}

\begin{abstract}
The matrices of spin-orbit interaction of the $d^{4} s$ configuration have been calculated. These matrices are for use in calculations to aid in locating levels and making electron configuration assignments in Te I and Ta I. The calculations were made by using methods and tables given by G. Racah and by the use of tables of the $W$-coefficients prepared by L. C. Biedenharn, and by Obi and coworkers. The matrices have been checked by calculating their eigenvalues with the National Bureau of Standards Electronic Computer (SEAC).
\end{abstract}

The problem of locating and classifying the energy levels of a particular atomic system is aided considerably if approximate values of the predicted levels can be determined. These approximate values are obtained by comparison with corresponding levels in analogous spectra and from calculations of the wave functions and energy levels by well-established perturbation methods [1] ${ }^{1}$ The first step is to set up the matrices of the important interactions; this is done most easily by using methods developed by Racah [2,3]. The elements of the matrices are linear combinations of a limited number of radial integrals, the most important ones being the Slater integrals, $R^{k}$, for the electrostatic interaction and the spin-orbit integrals $\zeta_{l}$. The second step, which is outside the scope of this paper, is to assign numerical values to these integrals, and then calculate the eigenvalues and eigenvectors of the matrices, which are the energy levels and wave functions of the atomic system, respectively.

Until recently, most calculations were carried out in an LS-coupling approximation, neglecting the spin-orbit interaction $[1,2,4]$. This was mainly because inclusion of the spinorbit interaction greatly increases the size of the matrices, and hand methods of calculating the eigenvalues and eigenvectors are ruled out for all but the simpler systems. However, electronic digital computers are becoming more available for carrying out these calculations $[5,6]$, so that there is now a use for the matrices of spin-orbit interaction.

These matrices are especially useful at the present time because theoretical studies of atomic spectra are mainly directed toward the spectra of the heavy elements and the rare earths, in which it would be a poor approximation to neglect spin-orbit interaction $[5,6,7,8]$. This theoretical interest follows the experimental interest in these spectra (in various laboratories throughout the world), which is largely directed toward obtaining material for inclusion in the National Bureau of Standards series of atomic energy levels publications [9]. The present work was carried out to aid the experimental analyses of the first spectra of technetium and tantalum, currently being carried out for inclusion in the third volume of the publication.

In table 1, the matrices associated with the spin-orbit parameter $\zeta_{l}$ are set up for the $d^{4} s$ configuration. The rows and columns of the matrices are specified by the name of the $L S$-coupling state, using the usual spectroscopic notation. In addition, a prefixed subscript is added to the designation of the parent term in $d^{4}$. This is the seniority number and is used to distinguish states of the same $S$ and $L$ values in this configuration (the concept of seniority number, and this notation, is explained in reference 3). The methods of Racah were used in calculating these matrices $[2,3]$ (the explicit formula has been given by Trees in footnote 9 of reference 4). Tables of the $W$-coefficients, needed for this calculation, have been prepared by Biedenharn [10] and by Obi and his coworkers [11]. The matrices have been checked by calculating their eigenvalues with the National Bureau of Standards digital computer (SEAC), and comparing them with the correct values known from $j j$-coupling theory (the latter theory is outlined in chapter 10 of reference 1 ).

${ }^{1}$ Figures in brackets indicate the literature references on page 96. 
[1] E. U. Condon and G. H. Shortley, The theory of atomic spectra (Cambridge University Press, London, 1951).

[2] G. Racah, Phys. Rev. 62, 438 (1942).

[3] G. Racah, Phys. Rev. 63, 367 (1943).

[4] R. E. Trees, Phys. Rev. 83, 756 (1951).

[5] G. Racah, Lund Lecture, Proc. Rydberg Centennial Conference on Atomic Spectroscopy, Kungl. Fys. Sällskap. Handl. N. F. 65, 31 (1955).

[6] R. E. Trees, W. F. Cahill, P. Rabinowitz, J. Research NBS 55, 335 (1955) RP2639.

[7] M. Gehatiah, Phys. Rev. 94, 618 (1954).

[8] D. Sperber, Bul. Research Council Israel III, 437 (1954).

[9] C. E. Moore, Atomic energy levels, Cir. 467, National Bureau of Standards, vol. I (1949), vol. II (1952).

[10] L. C. Biedenharn, Tables of the Racah coefficients, Oak Ridge Nat. Lab., ORNL-1098 (1952).

[11] Obi, Ishidzu, Horie, Yanagawa, Tanabe, and Sato, Tables of the Racah coefficients, Annals of the Tokyo Astronomical Observatory, Second Series III, No. 3 (1953); IV, No. 1 (1954).

TABLE 1. Matrices of spin-orbit interaction of the $d^{4} s$ configuration

(The unit of the element is $\zeta_{d}$ )

$J=1 / 2$

\begin{tabular}{|c|c|c|c|c|c|c|c|c|c|}
\hline & $\left({ }_{0}^{1} \mathbf{S}\right)^{2} \mathbf{S}$ & $\left({ }_{4}^{1} \mathbf{S}\right)^{2} \mathbf{S}$ & $\left({ }_{2}^{3} \mathrm{P}\right)^{2} \mathrm{P}$ & $\left({ }_{2}^{3} \mathrm{P}\right)^{4} \mathrm{P}$ & $\left.{ }_{4}^{3} \mathrm{P}\right)^{2} \mathrm{P}$ & $\left({ }_{4}^{3} \mathrm{P}\right)^{4} \mathrm{P}$ & $\left({ }_{4}^{3} \mathrm{D}\right){ }^{4} \mathrm{D}$ & $\left({ }_{4}^{5} \mathrm{D}\right){ }^{4} \mathrm{D}$ & $\left({ }_{4}^{5} \mathrm{D}\right){ }^{6} \mathrm{D}$ \\
\hline$\left({ }_{0}^{1} \mathrm{~S}\right)^{2} \mathrm{~S}$ & 0 & 0 & $-\sqrt{3}$ & $-\sqrt{6}$ & 0 & 0 & 0 & 0 & 0 \\
\hline$\left({ }_{4}^{1} \mathrm{~S}\right)^{2} \mathrm{~S}$ & 0 & 0 & $+\frac{\sqrt{7}}{3}$ & $+\frac{\sqrt{14}}{3}$ & $-\frac{2 \sqrt{2}}{3}$ & $-\frac{4}{3}$ & 0 & 0 & 0 \\
\hline$\left({ }_{2}^{3} \mathrm{P}\right)^{2} \mathrm{P}$ & $-\sqrt{3}$ & $+\frac{\sqrt{7}}{3}$ & $-\frac{2}{9}$ & $-\frac{\sqrt{2}}{18}$ & $+\frac{4 \sqrt{14}}{9}$ & $+\frac{2 \sqrt{7}}{9}$ & 0 & $+\frac{2 \sqrt{10}}{3}$ & 0 \\
\hline$\left({ }_{2}^{3} \mathrm{P}\right){ }^{4} \mathrm{P}$ & $-\sqrt{6}$ & $+\frac{\sqrt{14}}{3}$ & $-\frac{\sqrt{2}}{18}$ & $-\frac{5}{18}$ & $+\frac{2 \sqrt{7}}{9}$ & $+\frac{5 \sqrt{14}}{9}$ & 0 & $+\frac{2 \sqrt{5}}{15}$ & $+\frac{2 \sqrt{30}}{5}$ \\
\hline$\left({ }_{4}^{3} \mathrm{P}\right)^{2} \mathrm{P}$ & 0 & $-\frac{2 \sqrt{2}}{3}$ & $+\frac{4 \sqrt{14}}{9}$ & $+\frac{2 \sqrt{7}}{9}$ & $-\frac{4}{9}$ & $-\frac{\sqrt{2}}{9}$ & $+\frac{\sqrt{3}}{2}$ & $-\frac{\sqrt{35}}{6}$ & 0 \\
\hline$\left({ }_{4}^{3} \mathrm{P}\right){ }^{4} \mathrm{P}$ & 0 & $-\frac{4}{3}$ & $+\frac{2 \sqrt{7}}{9}$ & $+\frac{5 \sqrt{14}}{9}$ & $-\frac{\sqrt{2}}{9}$ & $-\frac{5}{9}$ & $-\frac{\sqrt{6}}{4}$ & $-\frac{\sqrt{70}}{60}$ & $-\frac{\sqrt{105}}{10}$ \\
\hline$\left({ }_{4}^{3} \mathrm{D}\right)^{4} \mathrm{D}$ & 0 & 0 & 0 & 0 & $+\frac{\sqrt{3}}{2}$ & $-\frac{\sqrt{6}}{4}$ & $+\frac{1}{4}$ & $-\frac{\sqrt{105}}{20}$ & $+\frac{\sqrt{70}}{20}$ \\
\hline$\left({ }_{4}^{5} \mathrm{D}\right){ }^{4} \mathrm{D}$ & 0 & 0 & $+\frac{2 \sqrt{10}}{3}$ & $+\frac{2 \sqrt{5}}{15}$ & $-\frac{\sqrt{35}}{6}$ & $-\frac{\sqrt{70}}{60}$ & $-\frac{\sqrt{105}}{20}$ & $-\frac{27}{20}$ & $-\frac{\sqrt{6}}{20}$ \\
\hline$\left({ }_{4}^{5} \mathrm{D}\right){ }^{6} \mathrm{D}$ & 0 & 0 & 0 & $\frac{2 \sqrt{30}}{5}$ & 0 & $-\frac{\sqrt{105}}{10}$ & $+\frac{\sqrt{70}}{20}$ & $-\frac{\sqrt{6}}{20}$ & $-\frac{7}{5}$ \\
\hline
\end{tabular}


$J=3 / 2$

\begin{tabular}{|c|c|c|c|c|c|c|c|c|c|c|c|c|}
\hline & $\left({ }_{2}^{3} \mathrm{P}\right)^{2} \mathrm{P}$ & $\left({ }_{2}^{3} \mathrm{P}\right)^{4} \mathrm{P}$ & $\left({ }_{4}^{3} \mathrm{P}\right)^{2} \mathrm{P}$ & $\left({ }_{4}^{3} \mathrm{P}\right)^{4} \mathrm{P}$ & $\left({ }_{2}^{1} \mathrm{D}\right)^{2} \mathrm{D}$ & $\left({ }_{4}^{1} \mathrm{D}\right)^{2} \mathrm{D}$ & $\left({ }_{4}^{3} \mathrm{D}\right)^{2} \mathrm{D}$ & $\left({ }_{4}^{3} \mathrm{D}\right)^{4} \mathrm{D}$ & $\left({ }_{4}^{5} \mathrm{D}\right)^{4} \mathrm{D}$ & $\left({ }_{4}^{5} \mathrm{D}\right)^{6} \mathrm{D}$ & $\left({ }_{2}^{3} \mathrm{~F}\right)^{4} \mathrm{~F}$ & $\left({ }_{4}^{3} \mathrm{~F}\right)^{4} \mathrm{~F}$ \\
\hline$\left({ }_{2}^{3} \mathrm{P}\right)^{2} \mathrm{P}$ & $+\frac{1}{9}$ & $-\frac{\sqrt{5}}{18}$ & $-\frac{2 \sqrt{14}}{9}$ & $+\frac{\sqrt{70}}{9}$ & $-\frac{\sqrt{7}}{6}$ & $+\frac{\sqrt{14}}{3}$ & 0 & 0 & $+\frac{2 \sqrt{5}}{3}$ & 0 & 0 & 0 \\
\hline$\left({ }_{2}^{3} \mathrm{P}\right)^{4} \mathrm{P}$ & $-\frac{\sqrt{5}}{18}$ & $-\frac{1}{9}$ & $+\frac{\sqrt{70}}{9}$ & $+\frac{2 \sqrt{14}}{9}$ & $+\frac{\sqrt{35}}{30}$ & $-\frac{2 \sqrt{70}}{30}$ & 0 & 0 & $+\frac{8}{15}$ & $+\frac{2 \sqrt{21}}{5}$ & 0 & 0 \\
\hline$\left({ }_{4}^{3} \mathrm{P}\right)^{2} \mathrm{P}$ & $-\frac{2 \sqrt{14}}{9}$ & $+\frac{\sqrt{70}}{9}$ & $+\frac{2}{9}$ & $-\frac{\sqrt{5}}{9}$ & $+\frac{\sqrt{2}}{3}$ & $+\frac{1}{6}$ & $-\frac{\sqrt{6}}{2}$ & $+\frac{\sqrt{6}}{4}$ & $-\frac{\sqrt{70}}{12}$ & 0 & 0 & 0 \\
\hline$\left({ }_{4}^{3} \mathrm{P}\right)^{4} \mathrm{P}$ & $+\frac{\sqrt{70}}{9}$ & $+\frac{2 \sqrt{14}}{9}$ & $-\frac{\sqrt{5}}{9}$ & $-\frac{2}{9}$ & $-\frac{\sqrt{10}}{15}$ & $-\frac{\sqrt{5}}{30}$ & $-\frac{\sqrt{30}}{20}$ & $-\frac{\sqrt{30}}{5}$ & $-\frac{\sqrt{14}}{15}$ & $-\frac{7 \sqrt{6}}{20}$ & 0 & 0 \\
\hline$\left({ }_{2}^{1} \mathrm{D}\right)^{2} \mathrm{D}$ & $-\frac{\sqrt{7}}{6}$ & $+\frac{\sqrt{35}}{30}$ & $+\frac{\sqrt{2}}{3}$ & $-\frac{\sqrt{10}}{15}$ & 0 & 0 & $-\frac{2 \sqrt{3}}{3}$ & $+\frac{2 \sqrt{3}}{3}$ & 0 & 0 & $-\frac{2 \sqrt{15}}{15}$ & $-\frac{4 \sqrt{15}}{15}$ \\
\hline$\left({ }_{4}^{1} \mathrm{D}\right)^{2} \mathrm{D}$ & $+\frac{\sqrt{14}}{3}$ & $-\frac{2 \sqrt{70}}{30}$ & $+\frac{1}{6}$ & $-\frac{\sqrt{5}}{30}$ & 0 & 0 & $+\frac{\sqrt{6}}{6}$ & $-\frac{\sqrt{6}}{6}$ & 0 & 0 & $-\frac{\sqrt{30}}{15}$ & $+\frac{4 \sqrt{30}}{15}$ \\
\hline$\left({ }_{4}^{3} \mathrm{D}\right)^{2} \mathrm{D}$ & 0 & 0 & $-\frac{\sqrt{6}}{2}$ & $-\frac{\sqrt{30}}{20}$ & $-\frac{2 \sqrt{3}}{3}$ & $+\frac{\sqrt{6}}{6}$ & $+\frac{1}{6}$ & $+\frac{1}{12}$ & $+\frac{\sqrt{105}}{12}$ & 0 & $+\frac{\sqrt{5}}{3}$ & $+\frac{\sqrt{5}}{15}$ \\
\hline$\left({ }_{4}^{3} \mathrm{D}\right)^{4} \mathrm{D}$ & 0 & 0 & $+\frac{\sqrt{6}}{4}$ & $-\frac{\sqrt{30}}{5}$ & $+\frac{2 \sqrt{3}}{3}$ & $-\frac{\sqrt{6}}{6}$ & $+\frac{1}{12}$ & $+\frac{1}{6}$ & $-\frac{\sqrt{105}}{30}$ & $+\frac{7 \sqrt{5}}{20}$ & $-\frac{\sqrt{5}}{3}$ & $-\frac{\sqrt{5}}{15}$ \\
\hline$\left({ }_{4}^{5} \mathrm{D}\right)^{4} \mathrm{D}$ & $+\frac{2 \sqrt{5}}{3}$ & $+\frac{8}{15}$ & $-\frac{\sqrt{70}}{12}$ & $-\frac{\sqrt{14}}{15}$ & 0 & 0 & $+\frac{\sqrt{105}}{12}$ & $-\frac{\sqrt{105}}{30}$ & $-\frac{9}{10}$ & $-\frac{\sqrt{21}}{20}$ & $-\frac{\sqrt{21}}{15}$ & $+\frac{\sqrt{21}}{15}$ \\
\hline$\left({ }_{4}^{5} \mathrm{D}\right)^{6} \mathrm{D}$ & 0 & $+\frac{2 \sqrt{21}}{5}$ & 0 & $-\frac{7 \sqrt{6}}{20}$ & 0 & 0 & 0 & $+\frac{7 \sqrt{5}}{20}$ & $-\frac{\sqrt{21}}{20}$ & $-\frac{11}{10}$ & $+\frac{1}{5}$ & $-\frac{1}{5}$ \\
\hline$\left({ }_{2}^{3} \mathrm{~F}\right)^{4} \mathrm{~F}$ & 0 & 0 & 0 & 0 & $-\frac{2 \sqrt{15}}{15}$ & $-\frac{\sqrt{30}}{15}$ & $+\frac{\sqrt{5}}{3}$ & $-\frac{\sqrt{5}}{3}$ & $-\frac{\sqrt{21}}{15}$ & $+\frac{1}{5}$ & $-\frac{2}{3}$ & $+\frac{2}{3}$ \\
\hline$\left({ }_{4}^{3} \mathrm{~F}\right)^{4} \mathrm{~F}$ & 0 & 0 & 0 & 0 & $-\frac{4 \sqrt{15}}{15}$ & $+\frac{4 \sqrt{30}}{15}$ & $+\frac{\sqrt{5}}{15}$ & $-\frac{\sqrt{5}}{15}$ & $+\frac{\sqrt{21}}{15}$ & $-\frac{1}{5}$ & $+\frac{2}{3}$ & $+\frac{1}{3}$ \\
\hline
\end{tabular}


$J=5 / 2$

\begin{tabular}{|c|c|c|c|c|c|c|c|c|c|c|c|c|c|c|}
\hline & $\left({ }_{2}^{3} \mathrm{P}\right)^{4} \mathrm{P}$ & $\left({ }_{4}^{3} \mathrm{P}\right)^{4} \mathrm{P}$ & $\left({ }_{2}^{1} \mathrm{D}\right)^{2} \mathrm{D}$ & $\left({ }_{4}^{1} \mathrm{D}\right)^{2} \mathrm{D}$ & $\left({ }_{4}^{3} \mathrm{D}\right)^{2} \mathrm{D}$ & $\left({ }_{4}^{3} \mathrm{D}\right)^{4} \mathrm{D}$ & $\left({ }_{4}^{5} \mathrm{D}\right)^{4} \mathrm{D}$ & $\left({ }_{4}^{5} \mathrm{D}\right)^{6} \mathrm{D}$ & $\left({ }_{4}^{1} \mathrm{~F}\right)^{2} \mathrm{~F}$ & $\left({ }_{2}^{3} \mathrm{~F}\right)^{2} \mathrm{~F}$ & $\left({ }_{2}^{3} \mathrm{~F}\right)^{4} \mathrm{~F}$ & $\left({ }_{4}^{3} \mathrm{~F}\right)^{2} \mathrm{~F}$ & $\left({ }_{4}^{3} \mathrm{~F}\right)^{4} \mathrm{~F}$ & $\left({ }_{4}^{3} \mathrm{G}\right)^{4} \mathrm{G}$ \\
\hline$\left({ }_{2}^{3} \mathrm{P}\right)^{4} \mathrm{P}$ & $+\frac{1}{6}$ & $-\frac{\sqrt{14}}{3}$ & $+\frac{\sqrt{210}}{30}$ & $-\frac{2 \sqrt{105}}{15}$ & 0 & 0 & $+\frac{2 \sqrt{21}}{15}$ & $+\frac{4 \sqrt{21}}{15}$ & 0 & 0 & 0 & 0 & 0 & 0 \\
\hline$\left({ }_{4}^{3} \mathrm{P}\right)^{4} \mathrm{P}$ & $-\frac{\sqrt{14}}{3}$ & $+\frac{1}{3}$ & $-\frac{2 \sqrt{15}}{15}$ & $-\frac{\sqrt{30}}{30}$ & $-\frac{3 \sqrt{5}}{10}$ & $-\frac{3 \sqrt{70}}{20}$ & $-\frac{7 \sqrt{6}}{60}$ & $-\frac{7 \sqrt{6}}{30}$ & 0 & 0 & 0 & 0 & 0 & 0 \\
\hline$\left({ }_{2}^{1} \mathrm{D}\right)^{2} \mathrm{D}$ & $+\frac{\sqrt{210}}{30}$ & $-\frac{2 \sqrt{15}}{15}$ & 0 & 0 & $+\frac{4 \sqrt{3}}{9}$ & $+\frac{2 \sqrt{42}}{9}$ & 0 & 0 & 0 & $-\frac{2 \sqrt{3}}{9}$ & $-\frac{4 \sqrt{15}}{45}$ & $-\frac{4 \sqrt{3}}{9}$ & $-\frac{8 \sqrt{15}}{45}$ & 0 \\
\hline$\left({ }_{4}^{1} \mathrm{D}\right)^{2} \mathrm{D}$ & $-\frac{2 \sqrt{105}}{15}$ & $-\frac{\sqrt{30}}{30}$ & 0 & 0 & $-\frac{\sqrt{6}}{9}$ & $-\frac{\sqrt{21}}{9}$ & 0 & 0 & 0 & $-\frac{\sqrt{6}}{9}$ & $-\frac{2 \sqrt{30}}{45}$ & $+\frac{4 \sqrt{6}}{9}$ & $+\frac{8 \sqrt{30}}{45}$ & 0 \\
\hline$\left({ }_{4}^{3} \mathrm{D}\right)^{2} \mathrm{D}$ & 0 & $-\frac{3 \sqrt{5}}{10}$ & $+\frac{4 \sqrt{3}}{9}$ & $-\frac{\sqrt{6}}{9}$ & $-\frac{1}{9}$ & $+\frac{\sqrt{14}}{36}$ & $+\frac{7 \sqrt{30}}{36}$ & 0 & $-\frac{2 \sqrt{15}}{9}$ & $-\frac{10}{9}$ & $+\frac{2 \sqrt{5}}{9}$ & $-\frac{2}{9}$ & $+\frac{2 \sqrt{5}}{45}$ & 0 \\
\hline$\left({ }_{4}^{3} \mathrm{D}\right)^{4} \mathrm{D}$ & 0 & $-\frac{3 \sqrt{70}}{20}$ & $+\frac{2 \sqrt{42}}{9}$ & $-\frac{\sqrt{21}}{9}$ & $+\frac{\sqrt{14}}{36}$ & $+\frac{1}{36}$ & $-\frac{\sqrt{105}}{180}$ & $+\frac{\sqrt{105}}{10}$ & $+\frac{2 \sqrt{210}}{63}$ & $-\frac{5 \sqrt{14}}{63}$ & $-\frac{8 \sqrt{70}}{63}$ & $-\frac{\sqrt{14}}{63}$ & $-\frac{8 \sqrt{70}}{315}$ & 0 \\
\hline$\left({ }_{4}^{5} \mathrm{D}\right)^{4} \mathrm{D}$ & $+\frac{2 \sqrt{21}}{15}$ & $-\frac{7 \sqrt{6}}{60}$ & 0 & 0 & $+\frac{7 \sqrt{30}}{36}$ & $-\frac{\sqrt{105}}{180}$ & $-\frac{3}{20}$ & $-\frac{3}{10}$ & 0 & $+\frac{\sqrt{30}}{9}$ & $-\frac{8 \sqrt{6}}{45}$ & $-\frac{\sqrt{30}}{9}$ & $+\frac{8 \sqrt{6}}{45}$ & 0 \\
\hline$\left({ }_{4}^{5} \mathrm{D}\right)^{6} \mathrm{D}$ & $+\frac{4 \sqrt{21}}{15}$ & $-\frac{7 \sqrt{6}}{30}$ & 0 & 0 & 0 & $+\frac{\sqrt{105}}{10}$ & $-\frac{3}{10}$ & $-\frac{3}{5}$ & 0 & 0 & $+\frac{\sqrt{6}}{5}$ & 0 & $-\frac{\sqrt{6}}{5}$ & 0 \\
\hline$\left({ }_{4}^{1} \mathrm{~F}\right)^{2} \mathrm{~F}$ & 0 & 0 & 0 & 0 & $-\frac{2 \sqrt{15}}{9}$ & $+\frac{2 \sqrt{210}}{63}$ & 0 & 0 & 0 & $-\frac{2 \sqrt{15}}{9}$ & $+\frac{5 \sqrt{3}}{9}$ & $-\frac{\sqrt{15}}{9}$ & $+\frac{5 \sqrt{3}}{18}$ & $+\frac{3 \sqrt{21}}{14}$ \\
\hline$\left({ }_{2}^{3} \mathrm{~F}\right)^{2} \mathrm{~F}$ & 0 & 0 & $-\frac{2 \sqrt{3}}{9}$ & $-\frac{\sqrt{6}}{9}$ & $-\frac{10}{9}$ & $-\frac{5 \sqrt{14}}{63}$ & $+\frac{\sqrt{30}}{9}$ & 0 & $-\frac{2 \sqrt{15}}{9}$ & $-\frac{4}{9}$ & $-\frac{\sqrt{5}}{9}$ & $+\frac{4}{9}$ & $+\frac{\sqrt{5}}{9}$ & $+\frac{\sqrt{35}}{7}$ \\
\hline$\left({ }_{2}^{3} \mathrm{~F}\right)^{4} \mathrm{~F}$ & 0 & 0 & $-\frac{4 \sqrt{15}}{45}$ & $-\frac{2 \sqrt{30}}{45}$ & $+\frac{2 \sqrt{5}}{9}$ & $-\frac{8 \sqrt{70}}{63}$ & $-\frac{8 \sqrt{6}}{45}$ & $+\frac{\sqrt{6}}{5}$ & $+\frac{5 \sqrt{3}}{9}$ & $-\frac{\sqrt{5}}{9}$ & $\frac{7}{18}$ & $+\frac{\sqrt{5}}{9}$ & $+\frac{7}{18}$ & $-\frac{5 \sqrt{7}}{14}$ \\
\hline$\left({ }_{4}^{3} \mathrm{~F}\right)^{2} \mathrm{~F}$ & 0 & 0 & $-\frac{4 \sqrt{3}}{9}$ & $+\frac{4 \sqrt{6}}{9}$ & $-\frac{2}{9}$ & $-\frac{\sqrt{14}}{63}$ & $-\frac{\sqrt{30}}{9}$ & 0 & $-\frac{\sqrt{ } 1 \overline{5}}{9}$ & $+\frac{4}{9}$ & $+\frac{\sqrt{5}}{9}$ & $+\frac{2}{9}$ & $+\frac{\sqrt{5}}{18}$ & $-\frac{\sqrt{35}}{14}$ \\
\hline$\left({ }_{4}^{3} \mathrm{~F}\right)^{4} \mathrm{~F}$ & 0 & 0 & $-\frac{8 \sqrt{15}}{45}$ & $+\frac{8 \sqrt{30}}{45}$ & $+\frac{2 \sqrt{5}}{45}$ & $-\frac{8 \sqrt{70}}{315}$ & $+\frac{8 \sqrt{6}}{45}$ & $-\frac{\sqrt{6}}{5}$ & $+\frac{5 \sqrt{3}}{18}$ & $+\frac{\sqrt{5}}{9}$ & $+\frac{7}{18}$ & $+\frac{\sqrt{5}}{18}$ & $+\frac{7}{36}$ & $+\frac{5 \sqrt{7}}{28}$ \\
\hline$\left({ }_{4}^{3} \mathrm{G}\right)^{4} \mathrm{G}$ & 0 & 0 & 0 & 0 & 0 & 0 & 0 & 0 & $+\frac{3 \sqrt{21}}{14}$ & $+\frac{\sqrt{35}}{7}$ & $-\frac{5 \sqrt{7}}{14}$ & $-\frac{\sqrt{35}}{14}$ & $+\frac{5 \sqrt{7}}{28}$ & $-\frac{3}{4}$ \\
\hline
\end{tabular}




\begin{tabular}{|c|c|c|c|c|c|c|c|c|c|c|c|c|c|}
\hline & $\left({ }_{4}^{3} \mathrm{D}\right)^{4} \mathrm{D}$ & $\left({ }_{4}^{5} \mathrm{D}\right)^{4} \mathrm{D}$ & $\left({ }_{14}^{5} \mathrm{D}\right)^{6} \mathrm{D}$ & $\left({ }_{4}^{1} \mathrm{~F}\right)^{2} \mathrm{~F}$ & $\left({ }_{2}^{3} \mathrm{~F}\right)^{2} \mathrm{~F}$ & $\left({ }_{2}^{3} \mathrm{~F}\right)^{4} \mathrm{~F}$ & $\left({ }_{4}^{3} \mathrm{~F}\right)^{2} \mathrm{~F}$ & $\left({ }_{4}^{3} \mathrm{~F}\right)^{4} \mathrm{~F}$ & $\left({ }_{2}^{1} G\right)^{2} \mathrm{G}$ & $\left({ }_{4}^{1} \mathrm{G}\right)^{2} \mathrm{G}$ & $\left({ }_{4}^{3} \mathrm{G}\right)^{2} \mathrm{G}$ & $\left({ }_{4}^{3} \mathrm{G}\right)^{4} \mathrm{G}$ & $\left({ }_{4}^{3} \mathrm{H}\right)^{4} \mathrm{H}$ \\
\hline$\left({ }_{4}^{3} \mathrm{D}\right)^{4} \mathrm{D}$ & $-\frac{1}{6}$ & $+\frac{\sqrt{105}}{30}$ & $+\frac{\sqrt{105}}{10}$ & $+\frac{2 \sqrt{105}}{21}$ & $-\frac{5 \sqrt{7}}{21}$ & $-\frac{5 \sqrt{21}}{21}$ & $-\frac{\sqrt{7}}{21}$ & $-\frac{\sqrt{21}}{21}$ & 0 & 0 & 0 & 0 & 0 \\
\hline$\left({ }_{4}^{5} \mathrm{D}\right)^{4} \mathrm{D}$ & $+\frac{\sqrt{105}}{30}$ & $+\frac{9}{10}$ & $-\frac{3}{10}$ & 0 & $+\frac{\sqrt{15}}{3}$ & $-\frac{\sqrt{5}}{5}$ & $-\frac{\sqrt{15}}{3}$ & $+\frac{\sqrt{5}}{5}$ & 0 & 0 & 0 & 0 & 0 \\
\hline$\left({ }_{4}^{5} \mathrm{D}\right)^{6} \mathrm{D}$ & $+\frac{\sqrt{105}}{10}$ & $-\frac{3}{10}$ & $+\frac{1}{10}$ & 0 & 0 & $+\frac{2 \sqrt{5}}{5}$ & 0 & $-\frac{2 \sqrt{5}}{5}$ & 0 & 0 & 0 & 0 & 0 \\
\hline$\left({ }_{4}^{1} F\right)^{2} F$ & $+\frac{2 \sqrt{105}}{21}$ & 0 & 0 & 0 & $+\frac{\sqrt{15}}{6}$ & $+\frac{\sqrt{5}}{2}$ & $+\frac{\sqrt{15}}{12}$ & $+\frac{\sqrt{5}}{4}$ & 0 & 0 & $+\frac{3}{4}$ & $+\frac{3 \sqrt{35}}{28}$ & 0 \\
\hline$\left({ }_{2}^{3} \mathrm{~F}\right)^{2} \mathrm{~F}$ & $-\frac{5 \sqrt{7}}{21}$ & $+\frac{\sqrt{15}}{3}$ & 0 & $+\frac{\sqrt{15}}{6}$ & $+\frac{1}{3}$ & $-\frac{\sqrt{3}}{6}$ & $-\frac{1}{3}$ & $+\frac{\sqrt{3}}{6}$ & $-\frac{\sqrt{3}}{6}$ & $+\frac{\sqrt{33}}{6}$ & $-\frac{\sqrt{15}}{3}$ & $+\frac{5 \sqrt{21}}{42}$ & 0 \\
\hline$\left({ }_{2}^{3} \mathrm{~F}\right)^{4} \mathrm{~F}$ & $-\frac{5 \sqrt{21}}{21}$ & $-\frac{\sqrt{5}}{5}$ & $+\frac{2 \sqrt{5}}{5}$ & $+\frac{\sqrt{5}}{2}$ & $-\frac{\sqrt{3}}{6}$ & 0 & $+\frac{\sqrt{3}}{6}$ & 0 & $+\frac{1}{6}$ & $-\frac{\sqrt{11}}{6}$ & $-\frac{\sqrt{5}}{6}$ & $-\frac{10 \sqrt{7}}{21}$ & 0 \\
\hline$\left({ }_{4}^{3} \mathrm{~F}\right)^{2} \mathrm{~F}$ & $-\frac{\sqrt{7}}{21}$ & $-\frac{\sqrt{15}}{3}$ & 0 & $+\frac{\sqrt{15}}{12}$ & $-\frac{1}{3}$ & $+\frac{\sqrt{3}}{6}$ & $-\frac{1}{6}$ & $+\frac{\sqrt{3}}{12}$ & $+\frac{5 \sqrt{3}}{6}$ & $-\frac{\sqrt{3 \overline{3}}}{12}$ & $+\frac{\sqrt{15}}{6}$ & $-\frac{5 \sqrt{21}}{84}$ & 0 \\
\hline$\left({ }_{4}^{3} \mathrm{~F}\right)^{4} \mathrm{~F}$ & $-\frac{\sqrt{21}}{21}$ & $+\frac{\sqrt{5}}{5}$ & $-\frac{2 \sqrt{5}}{5}$ & $+\frac{\sqrt{5}}{4}$ & $+\frac{\sqrt{3}}{6}$ & 0 & $+\frac{\sqrt{3}}{12}$ & 0 & $-\frac{5}{6}$ & $+\frac{\sqrt{11}}{12}$ & $+\frac{\sqrt{5}}{12}$ & $+\frac{5 \sqrt{7}}{21}$ & 0 \\
\hline$\left({ }_{2}^{1} G\right)^{2} \mathrm{G}$ & 0 & 0 & 0 & 0 & $-\frac{\sqrt{3}}{6}$ & $+\frac{1}{6}$ & $+\frac{5 \sqrt{3}}{6}$ & $-\frac{5}{6}$ & 0 & 0 & $-\frac{\sqrt{5}}{6}$ & $+\frac{\sqrt{7}}{6}$ & $+\frac{\sqrt{22}}{3}$ \\
\hline$\left({ }_{4}^{1} \mathrm{G}\right)^{2} \mathrm{G}$ & 0 & 0 & 0 & 0 & $+\frac{\sqrt{33}}{6}$ & $-\frac{\sqrt{11}}{6}$ & $-\frac{\sqrt{33}}{12}$ & $+\frac{\sqrt{11}}{12}$ & 0 & 0 & $-\frac{\sqrt{55}}{12}$ & $+\frac{\sqrt{77}}{12}$ & $-\frac{2 \sqrt{2}}{3}$ \\
\hline$\left({ }_{4}^{3} \mathrm{G}\right)^{2} \mathrm{G}$ & 0 & 0 & 0 & $+\frac{3}{4}$ & $-\frac{\sqrt{15}}{3}$ & $-\frac{\sqrt{5}}{6}$ & $+\frac{\sqrt{15}}{6}$ & $+\frac{\sqrt{5}}{12}$ & $-\frac{\sqrt{5}}{6}$ & $-\frac{\sqrt{55}}{12}$ & $-\frac{1}{2}$ & $-\frac{\sqrt{35}}{20}$ & $+\frac{\sqrt{110}}{15}$ \\
\hline$\left({ }_{4}^{3} \mathrm{G}\right)^{4} \mathrm{G}$ & 0 & 0 & 0 & $+\frac{3 \sqrt{35}}{28}$ & $+\frac{5 \sqrt{21}}{42}$ & $-\frac{10 \sqrt{7}}{21}$ & $-\frac{5 \sqrt{21}}{84}$ & $+\frac{5 \sqrt{7}}{21}$ & $+\frac{\sqrt{7}}{6}$ & $+\frac{\sqrt{77}}{12}$ & $-\frac{\sqrt{35}}{20}$ & $-\frac{2}{5}$ & $-\frac{\sqrt{154}}{15}$ \\
\hline$\left({ }_{4}^{3} \mathrm{H}\right)^{4} \mathrm{H}$ & 0 & 0 & 0 & 0 & 0 & 0 & 0 & 0 & $+\frac{\sqrt{22}}{3}$ & $-\frac{2 \sqrt{2}}{3}$ & $+\frac{\sqrt{110}}{15}$ & $-\frac{\sqrt{154}}{15}$ & $-\frac{3}{5}$ \\
\hline
\end{tabular}




\begin{tabular}{|c|c|c|c|c|c|c|c|c|c|}
\hline & $\left({ }_{4}^{5} \mathrm{D}\right)^{6} \mathrm{D}$ & $\left({ }_{2}^{3} \mathrm{~F}\right)^{4} \mathrm{~F}$ & $\left({ }_{4}^{3} \mathrm{~F}\right)^{4} \mathrm{~F}$ & $\left({ }_{2}^{1} G\right)^{2} \mathrm{G}$ & $\left({ }_{4}^{1} \mathrm{G}\right)^{2} \mathrm{G}$ & $\left({ }_{4}^{3} \mathrm{G}\right)^{2} \mathrm{G}$ & $\left({ }_{4}^{3} \mathrm{G}\right)^{4} \mathrm{G}$ & $\left({ }_{4}^{3} \mathrm{H}\right)^{2} \mathrm{H}$ & $\left({ }_{4}^{3} \mathrm{H}\right)^{4} \mathrm{H}$ \\
\hline$\left({ }_{4}^{5} \mathrm{D}\right)^{6} \mathrm{D}$ & +1 & $+\sqrt{2}$ & $-\sqrt{2}$ & 0 & 0 & 0 & 0 & 0 & 0 \\
\hline$\left({ }_{2}^{3} \mathrm{~F}\right)^{4} \mathrm{~F}$ & $+\sqrt{2}$ & $+\frac{1}{2}$ & $-\frac{1}{2}$ & $+\frac{1}{3}$ & $-\frac{\sqrt{11}}{3}$ & $-\frac{\sqrt{5}}{3}$ & $-\frac{\sqrt{55}}{6}$ & 0 & 0 \\
\hline$\left({ }_{4}^{3} \mathrm{~F}\right)^{4} \mathrm{~F}$ & $-\sqrt{2}$ & $-\frac{1}{2}$ & $-\frac{1}{4}$ & $-\frac{5}{3}$ & $+\frac{\sqrt{11}}{6}$ & $+\frac{\sqrt{5}}{6}$ & $+\frac{\sqrt{55}}{12}$ & 0 & 0 \\
\hline$\left({ }_{2}^{1} \mathrm{G}\right)^{2} \mathrm{G}$ & 0 & $+\frac{1}{3}$ & $-\frac{5}{3}$ & 0 & 0 & $+\frac{2 \sqrt{5}}{15}$ & $+\frac{\sqrt{55}}{15}$ & $+\frac{\sqrt{330}}{15}$ & $+\frac{2 \sqrt{55}}{15}$ \\
\hline$\left({ }_{4}^{1} G\right)^{2} G$ & 0 & $-\frac{\sqrt{11}}{3}$ & $+\frac{\sqrt{11}}{6}$ & 0 & 0 & $+\frac{\sqrt{55}}{15}$ & $+\frac{11 \sqrt{5}}{30}$ & $-\frac{2 \sqrt{30}}{15}$ & $-\frac{4 \sqrt{5}}{15}$ \\
\hline$\left({ }_{4}^{3} \mathrm{G}\right)^{2} \mathrm{G}$ & 0 & $-\frac{\sqrt{5}}{3}$ & $+\frac{\sqrt{5}}{6}$ & $+\frac{2 \sqrt{5}}{15}$ & $+\frac{\sqrt{55}}{15}$ & $+\frac{2}{5}$ & $-\frac{\sqrt{11}}{10}$ & $-\frac{2 \sqrt{66}}{15}$ & $+\frac{2 \sqrt{11}}{15}$ \\
\hline$\left({ }_{4}^{3} \mathrm{G}\right)^{4} \mathrm{G}$ & 0 & $-\frac{\sqrt{55}}{6}$ & $+\frac{\sqrt{55}}{12}$ & $+\frac{\sqrt{55}}{15}$ & $+\frac{11 \sqrt{5}}{30}$ & $-\frac{\sqrt{11}}{10}$ & $+\frac{1}{20}$ & $-\frac{2 \sqrt{6}}{15}$ & $-\frac{16}{15}$ \\
\hline$\left({ }_{4}^{3} \mathrm{H}\right)^{2} \mathrm{H}$ & 0 & 0 & 0 & $+\frac{\sqrt{330}}{15}$ & $-\frac{2 \sqrt{30}}{15}$ & $-\frac{2 \sqrt{66}}{15}$ & $-\frac{2 \sqrt{6}}{15}$ & $-\frac{2}{5}$ & $-\frac{\sqrt{6}}{10}$ \\
\hline$\left({ }_{4}^{3} \mathrm{H}\right)^{4} \mathrm{H}$ & 0 & 0 & 0 & $+\frac{2 \sqrt{55}}{15}$ & $-\frac{4 \sqrt{5}}{15}$ & $+\frac{2 \sqrt{11}}{15}$ & $-\frac{16}{15}$ & $-\frac{\sqrt{6}}{10}$ & $-\frac{3}{10}$ \\
\hline
\end{tabular}

\begin{tabular}{|c|c|c|c|c|}
\hline & $J=11 / 2$ \\
\hline & $\left.{ }_{4}^{3} \mathrm{G}\right)^{4} \mathrm{G}$ & $\left({ }_{4}^{3} \mathrm{H}\right)^{2} \mathrm{H}$ & $\left({ }_{4}^{3} \mathrm{H}\right)^{4} \mathrm{H}$ & $\left({ }_{4}^{1} \mathrm{I}\right)^{2} \mathrm{I}$ \\
\hline$\left.{ }_{4}^{3} \mathrm{G}\right)^{4} \mathrm{G}$ & $+\frac{3}{5}$ & $-\frac{\sqrt{10}}{5}$ & $-\frac{\sqrt{26}}{5}$ & 0 \\
\hline$\left.{ }^{(3} \mathrm{H}\right)^{2} \mathrm{H}$ & $-\frac{\sqrt{10}}{5}$ & $+\frac{1}{3}$ & $-\frac{\sqrt{65}}{30}$ & $-\frac{\sqrt{39}}{6}$ \\
\hline$\left.{ }_{4}^{3} \mathrm{H}\right)^{4} \mathrm{H}$ & $-\frac{\sqrt{26}}{5}$ & $-\frac{\sqrt{65}}{30}$ & $+\frac{1}{15}$ & $+\frac{\sqrt{15}}{6}$ \\
\hline$-\left({ }_{4}^{1} \mathrm{I}\right)^{2} \mathrm{I}$ & 0 & $-\frac{\sqrt{39}}{6}$ & $+\frac{\sqrt{15}}{6}$ & 0 \\
\hline
\end{tabular}

\begin{tabular}{|c|c|c|}
\hline \multicolumn{3}{c}{$J=13 / 2$} \\
\hline & $\left.{ }_{4}^{3} \mathrm{H}\right)^{4} \mathrm{H}$ & $\left({ }_{4}^{1} \mathrm{I}\right)^{2} \mathrm{I}$ \\
\hline$\left.{ }_{4}^{3} \mathrm{H}\right)^{4} \mathrm{H}$ & $+\frac{1}{2}$ & $+\frac{\sqrt{6}}{2}$ \\
\hline$\left.{ }_{(4}^{1} \mathrm{I}\right)^{2} \mathrm{I}$ & $+\frac{\sqrt{6}}{2}$ & 0 \\
\hline
\end{tabular}

Washington, September 12, 1956. 